\title{
Proportional Fairness for QoS Enhancement in IEEE 802.11e WLANs ${ }^{+}$
}

\author{
Jeng Farn Lee ${ }^{1,3}$, Wanjiun Liao ${ }^{1,2}$, and Meng Chang Chen ${ }^{3}$ \\ ${ }^{1}$ Department of Electrical Engineering, National Taiwan University, Taipei, Taiwan \\ ${ }^{2}$ Graudate Institute of Communication Engineering, National Taiwan University, Taipei, Taiwan \\ ${ }^{3}$ Institute of Information Science, Academia Sinica, Taipei, Taiwan \\ Email: wiliao@ntu.edu.tw
}

\begin{abstract}
In this paper, we study the proportional fairness problem in IEEE 802.11e wireless local area networks (WLANs). With 802.11e EDCA, only priority-based service is supported. Such priority-based service, while allowing differentiated service for flows of different priorities, cannot ensure service amount in proportion to their demands. This calls for weighted fair service to be supported by EDCA. In this paper, we propose a mechanism called Weighted Fair - EDCA (WF-EDCA) to provide proportional fairness for IEEE 802.11 WLANs. With WF-EDCA, weighted fair service among different access categories (ACs) is provided, and strict priority service can also be implemented. We then conduct simulations based on ns-2 to compare the performance of WF-EDCA and EDCA. The results show that WF-EDCA outperforms EDCA in terms of providing proportional fairness and strict priority service for IEEE 802.11 WLANs while retaining comparable total throughput.
\end{abstract}

\section{INTRODUCTION}

IEEE $802.11 \mathrm{e}$ [1] is a supplementary standard of 802.11 Medium Access Control (MAC) to provide QoS for different kinds of applications. With 802.11e EDCA, only priority-based service is supported. Priority-based protocols, while allowing service differentiation for flows of different priorities, cannot ensure minimum guaranteed or expected throughputs for these flows. To solve such problems, fair queuing is required. With fair queuing, different flows contending for a shared link can be allocated bandwidth in proportion to their "weights." The major challenge of fair queuing in WLANs is that service provisioning must be fully distributed. This renders existing centralized mechanisms inappropriate. So far, no mechanism has been designed for EDCA to provide proportional fairness service.

In this paper, we propose a new mechanism called Weighted Fair EDCA (WF-EDCA) for proportional fairness service in IEEE 802.11e EDCA systems. With WF-EDCA, weighted fair service among different access categories (ACs) is provided. The backoff intervals of $\mathrm{ACs}$ are determined according to the distributed scheduling discipline DFS [2]. WF-EDCA can be easily extended to provide strict priority service, under the constraint that proportional fairness is still maintained. We then conduct simulation based on ns- 2 to demonstrate the performance of WF-EDCA over 802.11e EDCA. The results show that WF-EDCA can provide strict priority and proportional fairness service for flows of different ACs based on their assigned weights, instead of the fixed priority service as in the original 802.11e EDCA.

\section{WEIGHTED FAIR - ENHANCED DISTRIBUTED CHANNEL ACCESS (WF-EDCA)}

In this section, we describe the proposed proportional fairness mechanism called WF-EDCA for IEEE 802.11 WLANs. With WF-EDCA, 802.11e EDCA is enhanced by using Distributed Fair Scheduling (DFS) [2] in each backoff entity such that weighted fair service is provided for flows of different $\mathrm{ACs}$. Each $\mathrm{AC}$ is also assigned a positive real number $\phi[A C]$ indicating the weight of sharing the channel capacity. The calculation of BI of the head of line packet is based on (1) according to DFS.

$$
B I_{i}=\left\lfloor\left\lfloor\text { Scaling_Factor } \times \frac{L_{i}}{\phi_{i}}\right\rfloor \times \rho\right\rfloor,
$$

where $L_{i}$ is the head of line frame size, and $\rho$ is a random variable uniformly distributed in $[0.9,1.1]$. In (1), the ratio between $L_{i}$ and $\phi_{i}$ is from the finish tag, and the scaling factor allows the choice of an appropriate scale for the virtual time in DFS.

Note that WF-EDCA can be easily extended to provide strict priority, in which frames with higher priority are transmitted before the lower-priority ones. To implement strict priority, the AIFS values of the lower priority must be set larger than that of the higher priority plus its maximum contention window, i.e.,

\footnotetext{
+ This work was supported partly by National Science Council under a Center Excellence Grant NSC93-2752-E-002-006-PAE, and in part by the National Science Council, Taiwan, under grant number NSC94-2218-E-002-059.
} 


$$
A I F S[j] \geq A I F S[i]+C W_{\max [i]} \text { if } \mathrm{j}>\mathrm{i}
$$

To sum up, in WF-EDCA, each AC is associated with a backoff entity and an EDCA parameter set, i.e., AIFS[AC], CWmin[AC], and $C W \max [\mathrm{AC}]$, but the value of the AIFS[AC] is now determined by (2). The backoff intervals of strict priority are the same as EDCA and that of proportional fairness are calculated based on (1).

\section{PERFORMANCE EVALUATION}

In this section, the faimess performance of the 802.11e EDCA and WF-EDCA is evaluated with the ns-2 EDCA implementation created by Chesson and Singla[3]. The wireless link rate is set at $11 \mathrm{Mbps}$ and each data flow is associated with one $\mathrm{AC}$ and generates CBR UDP traffic at a rate of $8 \mathrm{Mbps}$ to the AP. The packets, including the IP header, of all ACs are fixed at 1520 bytes. The weights of $\mathrm{AC} 0, \mathrm{AC} 1, \mathrm{AC} 2$ and $\mathrm{AC} 3$ are set to be $0.4,0.3,0.2$ and 0.1 , respectively, and the parameters Scaling_Factor of DFS are 0.01 in the simulations. The values of the EDCA parameter sets for the four ACs are listed in Table 1.

Table 1. Values of EDCA parameter sets used in the simulation

\begin{tabular}{|c|c|c|c|c|}
\hline & AC VO & AC VI & AC BE & AC BK \\
\hline AIFSN & 2 & 2 & 3 & 7 \\
\hline$C$ Wmin & 3 & 7 & 15 & 15 \\
\hline$C$ Wmax & 7 & 15 & 1023 & 1023 \\
\hline
\end{tabular}

We compare the fairness of $802.11 \mathrm{EDCA}$ and the proposed WF-EDCA for four different AC flows within five stations (STA) (Fig. 1 (a)). Fig. 1 plots the aggregate flow throughput of the same AC in the system for $802.11 \mathrm{e}$ EDCA and WF-EDCA (i.e., sub-figure (b) is for 802.11e EDCA and (c) for WF-EDCA). In the sub-figures, we identify the throughput ratios of $\mathrm{AC} 0, \mathrm{AC} 1, \mathrm{AC} 2$, and $\mathrm{AC} 3$ with respect to $\mathrm{AC} 3$. We observe that in all cases, with 802.11e EDCA, the high priority $\mathrm{AC}$ flows (i.e., $\mathrm{AC}$ 0) consumes most of the channel capacity, while the low priority ones (i.e., $\mathrm{AC} 2$ and $\mathrm{AC} 3$ ) are very likely to starve. WF-EDCA, on the other hand, allows AC flows to share channel capacity according to their assigned weights, thus providing proportional fairness nicely.

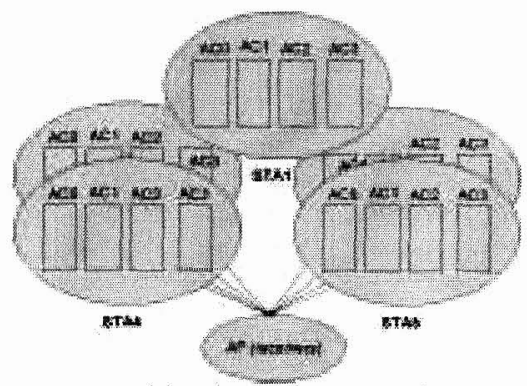

(a) simulation scenario

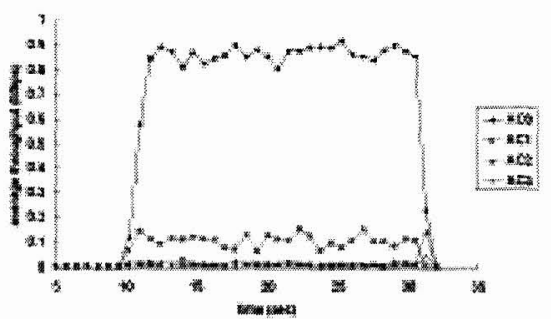

(b) $\mathrm{EDCA}(\mathrm{AC0}: \mathrm{AC1}: \mathrm{AC2}: \mathrm{AC} 3=176.95: 20.83: 3.4: 1)$

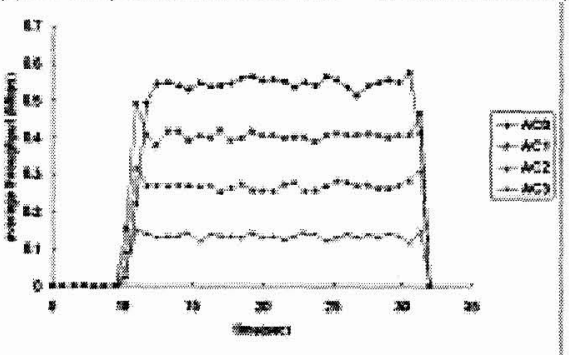

(c) WF-EDCA (AC0: AC1: $\mathrm{AC} 2: \mathrm{AC} 3=4.05: 3.04: 2.03: 1$ )

Figure 1. Aggregate throughput for four AC flows at five STAs

\section{CONCLUSION}

In this paper, we have proposed a proportional fairness service mechanism called Weighted Fair Enhanced Distributed Channel Access (WF-EDCA) for IEEE 802.11e WLANs. With WF-EDCA, weighted fair service among $\mathrm{ACs}$ is provided. The backoff intervals of ACs are determined according to the distributed scheduling discipline DFS. We have also demonstrated that WFEDCA can be easily extended to provide strict priority service, while proportional faimess is still maintained. We have then conducted simulations based on ns-2 and compared the performance of IEEE 802.11e EDCA and WF-EDCA in terms of proportional service, fairness, strict priority, and aggregate throughput of each AC. The simulation results show that with WF-EDCA, the ACs can share the channel capacity according to their assigned weights. In addition, WF-EDCA can provide strict priority service while maintaining the proportional fairness among different ACs. Most importantly, WF-EDCA is easily implemented and is compatible with the IEEE 802.11 Standard.

\section{REFERENCES}

[1] IEEE WG, Draft Supplement to Standard for Wireless LAN Medium Access Control (MAC) and Physical Layer (PHY) Specifications: Medium Access Control (MAC), Enhancements for Quality of Service (QoS), IEEE 802.11e Draft 4.I, Feb. 2003.

[2] N. H. Vaidya, P. Bahl, and S. Gupta, "Distributed Fair Scheduling in Wireless LAN," ACM Mobicom 2000.

[3] ns-2, 802.11e EDCA, available at http://wwwsop.inria.fr/planeteigni/Research.html 